\title{
Outcomes of anterior versus posterior peroral endoscopic myotomy 2 years post-procedure: prospective follow-up results from a randomized clinical trial
}

Authors

Yervant Ichkhanian ${ }^{1}$, Jad P. Abimansour ${ }^{1}$, Mathieu Pioche², Kia Vosoughi' ${ }^{1}$, Nicholas Eleftheriadis ${ }^{3,4}$, Philip Wai Yan Chiu $^{5}$, Hitomi Minami ${ }^{6}$, Kumi Ogihara ${ }^{6}$, Omid Sanaei ${ }^{1}$, Manol Jovani ${ }^{1}$, Mouen A. Khashab ${ }^{1}$

Institutions

1 Division of Gastroenterology and Hepatology, Johns Hopkins Medical Institutions, Baltimore, Maryland, United States

2 Endoscopy and Gastroenterology Unit, Edouard Herriot Hospital, Lyon, France

3 Endoscopy Department, Gastroenterology Unit, Metropolitan Hospital Athens, Athens, Greece

4 Medical School, Aristotle University of Thessaloniki, Thessaloniki, Greece

5 Division of Upper GI and Metabolic Surgery, Department of Surgery, The Chinese University of Hong Kong, Shatin, Hong Kong

6 Department of Gastroenterology and Hepatology, Nagazaki University Hospital, Nagazaki, Japan

submitted 5.4 .2020

accepted after revision 22.6.2020

published online 22.6.2020

Bibliography

Endoscopy 2021; 53: 462-468

DOI 10.1055/a-1204-4242

ISSN 0013-726X

(c) 2020. Thieme. All rights reserved.

Georg Thieme Verlag KG, Rüdigerstraße 14,

70469 Stuttgart, Germany

Corresponding author

Mouen A. Khashab, MD, Division of Gastroenterology and Hepatology, Johns Hopkins Hospital, Suite 7125G, Sheikh Zayed Bldg, 1800 Orleans Street, Baltimore, MD 21287, United States

Fax: +1-443-683-8335

mkhasha1@jhmi.edu

\section{ABSTRACT}

Background Peroral endoscopic myotomy (POEM) is considered a primary treatment modality for achalasia. It can be performed using either the anterior or posterior approach. A previous randomized clinical trial (RCT) showed that the posterior approach was noninferior to the anterior approach at 1 year post-POEM in terms of clinical success, rate of adverse event, and risk of gastroesophageal reflux disease (GERD). The aim of this post-RCT study was to compare outcomes at $\geq 2$ years post-POEM.

Methods Patients who previously completed the 1-year follow-up were contacted and their Eckardt, dysphagia, and GERD questionnaire (GERDQ) scores and frequency of proton pump inhibitor use were recorded. Clinical success was defined as an Eckardt score $<3$.

Results 150 patients were initially randomized and 138 completed the 1-year follow-up. Of the 138, 111 (anterior group 54, posterior group 57) also completed $\geq 2$ years of follow-up, with an overall clinical success decrease from $89 \%$ to $82 \%$. At $\geq 2$ years post-POEM, clinical success was achieved in $46 / 54(85 \%)$ and $45 / 57(79 \%)$ in the anterior and posterior groups, respectively $(P=0.43)$. A similar decrease in clinical success was noted in both groups at $\geq 2$ years (anterior: $90 \%$ to $85 \%$; posterior $89 \%$ to $79 \%$; $P=$ 0.47 ). GERDQ score was 6 (interquartile range $6-8 ; P=$ 0.08 ) in both treatment groups.

Conclusions The anterior and posterior POEM techniques remained equally effective at 2 years and decreases in efficacy were similar between the two approaches over time. GERD outcomes were also similar in both groups during medium-term follow-up. 

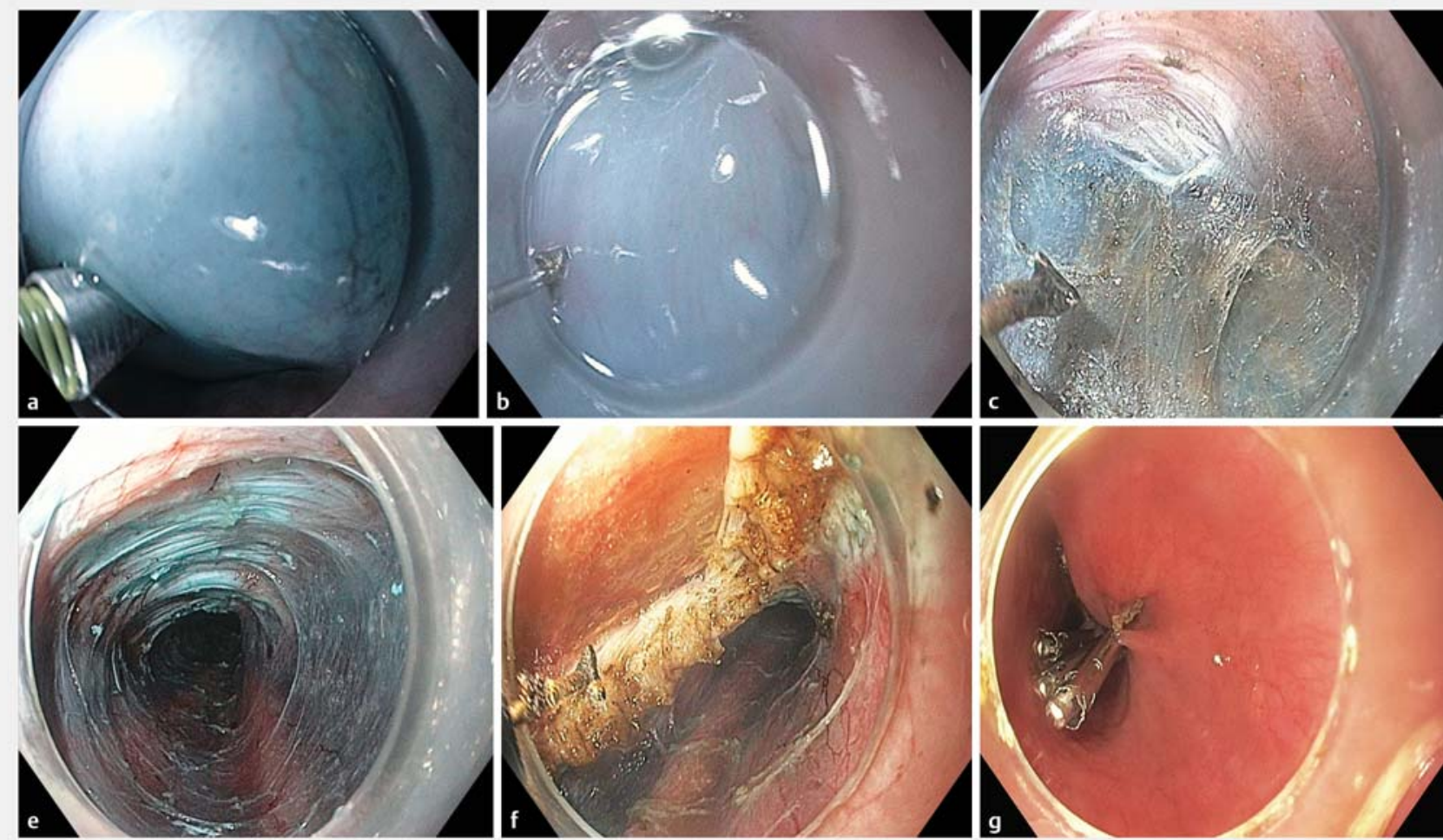

- Fig. 1 Peroral endoscopic myotomy, anterior approach (12 to 2 o'clock position), in a 45-year-old woman with type I achalasia. a, b Initially, the gastroscope was advanced to the mid-esophagus and a mucosal bleb was created by submucosal injection of mixed solution of $1 \%$ indigo carmine and saline. Using a triangular tip (TT) knife, a 1.5-cm mucosal incision was performed. c, d Following access to the submucosal space, spray coagulation ( $50 \mathrm{~W}$, effect 2 ) was utilized to dissect the submucosal fibers and create a submucosal tunnel. e The tunnel was extended $2 \mathrm{~cm}$ into the gastric cardia. Using the TT knife, a full-thickness myotomy was performed. f Following completion of the myotomy, the gastroscope was withdrawn from the submucosal tunnel and the mucosal incision site was secured with clips.

\section{Introduction}

Peroral endoscopic myotomy (POEM) is currently a well-established treatment for achalasia and has become a first-line management option at many high-volume centers [1-3]. Clinical and technical outcomes are comparable to conventional laparoscopic Heller myotomy [4]. The procedure utilizes submucosal tunneling to access the lower esophageal sphincter and is essentially the endoscopic equivalent of the surgical myotomy. The myotomy can be performed in an anterior or posterior orientation, which corresponds to the transaction of muscle fibers at 1 to 2 o'clock vs. 5 to 6 o'clock positions, respectively. Theoretically, the anterior approach limits pathologic reflux as it preserves the oblique fibers of the lower esophageal sphincter, whereas the posterior approach minimizes bleeding risk as it avoids major branches of the left gastric artery within the anterior submucosa.

In current clinical practice, myotomy orientation is largely driven by operator preference. In recent years, several studies have focused on comparing the two approaches, revealing similar efficacy $[5,6]$ and possibly lower risk of post-POEM gastroesophageal reflux with the posterior approach [6]. However, the limitation remains the lack of longitudinal data, as all comparative studies reported outcomes with a follow-up of less than 12 months.
We previously conducted a randomized clinical trial (RCT) of 150 patients to compare the two approaches and showed that the posterior approach was noninferior to the anterior approach, with no difference in clinical response, adverse events, or pathologic reflux at 1-year follow-up [7]. Comparative outcomes of both approaches during long-term follow-up are not currently known and are of clinical interest. The primary aim of the current study was to prospectively follow up a controlled, randomized cohort for at least 24 months post-POEM.

\section{Methods}

\section{Study design}

The study was a post-RCT (ClinicalTrials.gov NCT02454335) follow-up study. Patients who successfully completed 1 year of follow-up were contacted again at 2 years post-randomization to assess clinical success (Eckardt score $<3$ ), dysphagia score, and gastroesophageal reflux as measured by the gastroesophageal reflux disease questionnaire (GERDQ; a commonly used six-item subjective diagnostic and management tool [8]), and proton pump inhibitor (PPI) use.

The initial trial was a single-blinded, randomized, noninferiority study conducted at six tertiary referral hospitals (three Asia, two Europe, and one USA) from January 2015 to Novem- 


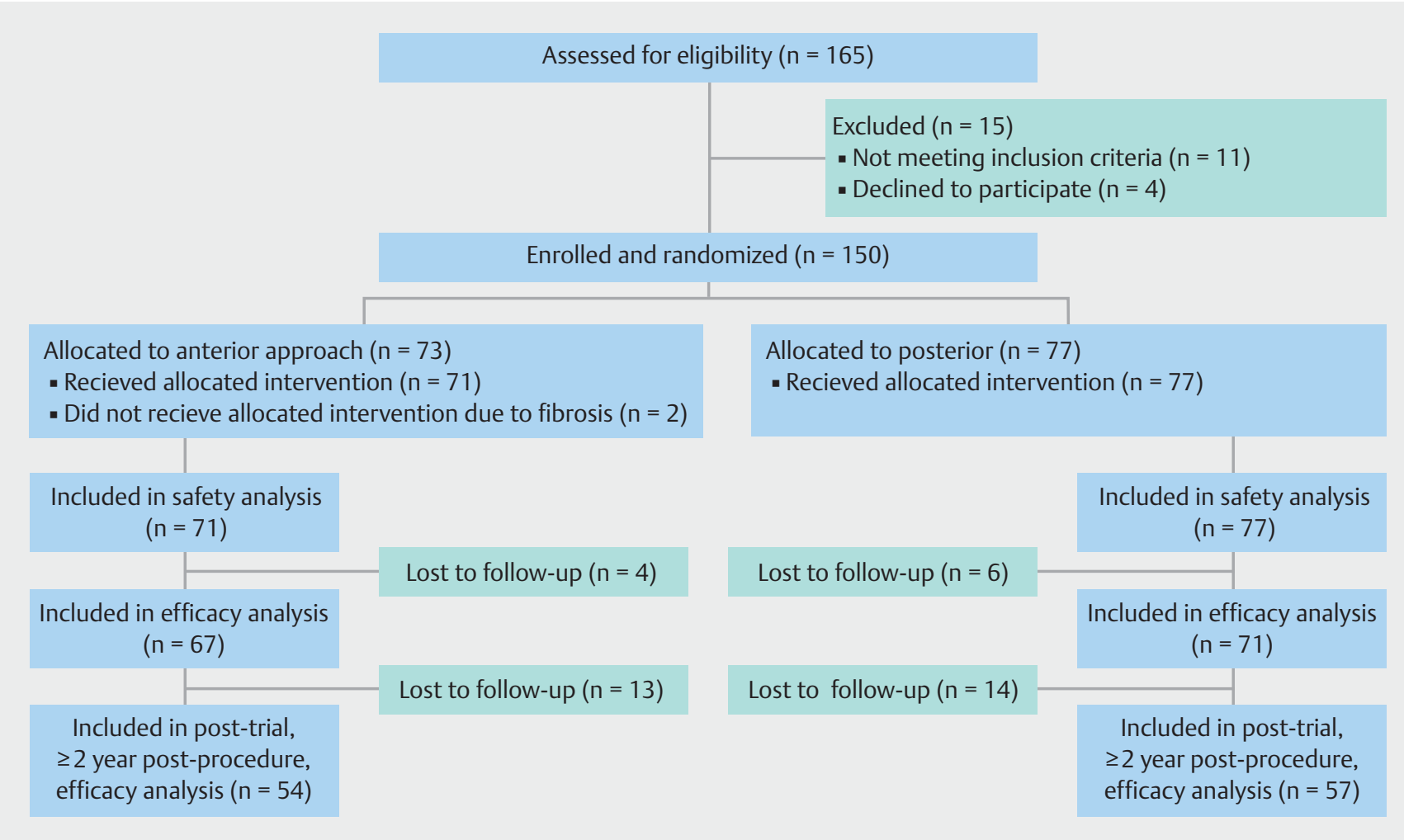

Fig. 2 Study flow diagram.

ber 2016 [7]. The trial was powered to detect noninferiority of a posterior approach compared with an anterior approach, which was generally considered the standard of practice. Patients diagnosed with achalasia on high-resolution esophageal manometry were randomly assigned in a 1:1 ratio to receive POEM with either the anterior or posterior approach ( $\mathbf{F i g . 1}$ ). A total of 138 patients successfully completed 1 year of follow-up [7].

For the current follow-up study, the 138 patients who completed the 1-year follow-up were contacted by the participating centers. All participating centers obtained approval from the institutional review board, or its equivalent, to conduct the follow-up study. A total of three attempts were made to contact patients via either a phone call or electronic mail. Patients who did not reply after the third attempt were recorded as lost to follow-up. The endoscopist or the research coordinator documented the follow-up results during either a phone call or a clinical encounter.

\section{End points}

The primary outcome was clinical success, defined as an Eckardt score $<3$ at least 2 years after the procedure. Secondary outcomes included dysphagia score, GERDQ score, and patient-reported use of PPIs (reported as none, occasional, daily).

\section{Statistical analysis}

Descriptive statistics were used to compare demographics as well as clinical and procedural characteristics between the two arms. Chi-squared testing was performed to compare the cate- gorical outcomes between the two groups, and student $t$ test and Mann-Whitney $U$ test were used to compare continuous variables. Logistic regression was performed to calculate the odds of PPI requirement at $\geq 2$ years post-POEM. Statistical significance was based on two-sided design-based tests evaluated at $\alpha=0.05$. Finally, across the time intervals, longitudinal data analyses of the clinical status (success vs. failure) and median GERDQ score were performed by incorporating all randomized patients and adjusting for dropout cases by fitting data in a mixed model. Statistical analyses were performed using SPSS software (version 17.0; SPSS Inc., Chicago Illinois, USA).

\section{Results}

In the initial trial [7] conducted between January 2015 and November 2017, 150 patients were randomized (anterior approach 73, posterior approach 77). A total of 138 patients completed the 1-year follow-up, with clinical success achieved in 89 $\%$ (90\% in the anterior group and $89 \%$ in the posterior group). There was no difference in dysphagia score (median score 0 , interquartile range [IQR] 0 in both groups) or GERDQ score (median score 6, IQR 6-8 in both groups). In the current study, a total of 111 patients ( $80 \%$; anterior group 54, posterior group 57) completed $\geq 2$ years of post-POEM follow-up ( $\mathbf{F i g . 2}$, see also Table $1 \mathrm{~s}$ in the online-only supplementary material). A total of 37 patients ( $25 \%$ ) who participated in the initial trial did not consent or were lost to follow-up. 
Table 1 Baseline demographic and procedural characteristics by treatment group.

\begin{tabular}{|c|c|c|c|}
\hline Variable & Anterior POEM & Posterior POEM & $P$ \\
\hline Total patients, n & 54 & 57 & \\
\hline Age, mean (SD), years & $52.3(21)$ & $51.2(18)$ & 0.6 \\
\hline Sex, female, n (\%) & $29(54)$ & $23(40)$ & 0.13 \\
\hline BMI, mean (SD), kg/m² & $24.5(4.5)$ & $23.3(3.5)$ & 0.32 \\
\hline \multicolumn{4}{|l|}{ Achalasia type, n (\%) } \\
\hline - Type I & $13(24)$ & $4(7)$ & 0.04 \\
\hline - Type II & $33(61)$ & $42(74)$ & \\
\hline - Type III & $8(15)$ & $11(19.3)$ & \\
\hline Symptom duration, mean (SD), months & $53.3(61.4)$ & $50.5(59.9)$ & 0.71 \\
\hline Hiatal hernia, n (\%) & $4(7)$ & $1(2)$ & 0.09 \\
\hline Baseline manometry pressure, mean (SD), mmHg & $38.3(23.2)$ & $37.2(15.3)$ & 0.23 \\
\hline Baseline relaxation residual pressure, mean (SD), $\mathrm{mmHg}$ & $28.3(12.5)$ & $27.2(8.1)$ & 0.34 \\
\hline \multicolumn{4}{|l|}{ Prior achalasia treatment, median (IQR) } \\
\hline - Botox toxin injection & $4(1-3)$ & $3.5(1-4)$ & 0.75 \\
\hline " Pneumatic dilation & $2(1-2)$ & $2(1-3.8)$ & 0.21 \\
\hline Anti-platelets/anti-coagulants, n (\%) & $9(17)$ & $5(9)$ & 0.95 \\
\hline Baseline pain, n (\%) & $21(39)$ & $20(35)$ & 0.63 \\
\hline ASA classification, mode & 2 & 2 & 0.24 \\
\hline \multicolumn{4}{|l|}{ Pre-POEM scores, median (IQR) } \\
\hline - Eckardt & $8(6-9)$ & $8(7-9)$ & 0.94 \\
\hline - Dysphagia & $2(1-3)$ & $3(1-3)$ & 0.35 \\
\hline - GERDQ & $9(6-11.5)$ & $10(8-12)$ & 0.12 \\
\hline Pre-POEM PPI use, n (\%) & $9(17)$ & $8(14)$ & 0.92 \\
\hline \multicolumn{4}{|l|}{ Length, mean (SD), cm } \\
\hline - Mucosal incision & $2.0(0.5)$ & $1.5(0.5)$ & 0.33 \\
\hline - Submucosal tunnel & $14.7(2.3)$ & $13.2(2.3)$ & 0.74 \\
\hline - Myotomy & $10.8(2.3)$ & $10.4(2.3)$ & 0.92 \\
\hline - Gastric myotomy & $3(1.3)$ & $2.8(2.10$ & 0.61 \\
\hline - Esophageal myotomy & $7.4(2.8)$ & $7.1(2.4)$ & 0.22 \\
\hline No. clips for incision closure, median (IQR) & $5(5-9)$ & $4.5(4-6)$ & 0.13 \\
\hline Total procedure duration, median (IQR), minutes & $63(49-101)$ & $64(46-107)$ & 0.11 \\
\hline Overall procedure difficulty, median (IQR)* & $2(1.5-3)$ & $2(1-2.5)$ & 0.15 \\
\hline
\end{tabular}


The two arms were equivalent at baseline, except for achalasia type and the presence of hernia ( Table 1). Achalasia type I, II, and III had frequencies of $13(24 \%), 33(61 \%)$, and $8(15 \%)$ in the anterior group, and $4(5 \%), 42(74 \%)$, and $11(19 \%)$ in the posterior group $(P=0.04)$. There was no significant difference in procedure-related characteristics, with median total procedure time of 63 (IQR 49-101) and 64 (IQR 46-107) minutes $(P=0.11)$ in the anterior and posterior group, respectively ( $\triangleright$ Table 1). In general, mean post-POEM follow-up time was 34.6 months (standard deviation [SD] 5.3; anterior arm 34.5 months [SD 6.9], posterior arm 32.5 months [SD 5.2]; $P=0.21$ ).

\section{Clinical outcomes}

Clinical success was achieved in $91 / 111$ patients ( $82 \%$ ) at $\geq 2$ years post-POEM. Specifically, clinical success was equivalent in the anterior and posterior groups (46/54 [85\%] vs. 45/57 [79\%]; $P=0.43)$. Binary regression analysis, controlling for achalasia subtype and presence of a hernia, did not reveal a significant difference between treatment groups in terms of clinical success. Clinical success declined between 1 year and $\geq 2$ years post-POEM, from $60 / 67$ (90\%) to $46 / 54$ (85\%) in the anterior group and from $63 / 71(89 \%)$ to $45 / 57(79 \%)$ in the posterior group $(P=0.47)$. The mean Eckardt score for patients who had clinical failure at $\geq 2$-year follow-up (anterior group 10, posterior group 10) was 5.8 (SD 2.2). The majority of these patients had a pre-POEM diagnosis of achalasia type II $(n=15,75$ $\%)$, followed by achalasia type I $(n=3,15 \%)$ and type III $(n=2$, $10 \%$ ) (Table $2 \mathrm{~s}$ ). Overall, the median dysphagia score at $\geq 2$ years was 0 (IQR 0$)$, with the majority of patients having a dysphagia score of 0 in both the anterior (79\%) and posterior (77\%) groups $(P=0.42)$. There was no change in the median dysphagia score between the 1 -year and $\geq 2$-year follow-up periods across both treatment arms $(P=0.71)$ ( $>$ Table 2$)$.

\section{Gastroesophageal reflux}

In both treatment groups, the median GERDQ score was 6 (IQR $6-8 ; P=0.08)$ at $\geq 2$ years post-POEM, with no worsening of the median GERDQ score when compared with 1 year. A total of 13 patients (24\%) in the anterior group and 12 $(21 \%)$ in the posterior group had GERDQ scores $>8(P=$ 0.22 ). For PPI usage, 76/111 (68\%) were off-PPI (anterior group 32 [59\%], posterior group 44 [77\%]; $P=0.07)$. In the anterior and the posterior groups, PPIs were take occasionally by $15(28 \%)$ and $8(14 \% ; P=0.09)$ patients, respectively, and daily by $7(13 \%)$ and $5(8.8 \% ; P=0.11)$ patients, respectively. There was a $13 \%$ increase in the rate of PPI use in the anterior group compared with the rate at 1 year post-POEM. Conversely, there was a $5 \%$ decrease in PPI use in the posterior group ( $\triangleright$ Table 2 ). Logistic regression analysis showed a nonsignificant tendency towards less PPI use at $\geq 2$ years post-POEM in the posterior group (odds ratio [OR] 0.4, $95 \%$ confidence interval $[\mathrm{Cl}] 0.17-1.04 ; P=0.06)$.

\section{Subgroup analysis by achalasia subtype}

Clinical success declined from $16 / 18(89 \%)$ at 1 year to $14 / 17$ $(82 \%)$ at $\geq 2$ years in patients with achalasia type I $(P=0.13)$, $88 / 98(90 \%)$ to $63 / 75(84 \%)$ in patients with achalasia type II
- Table 2 Changes in patients who completed both 1 and $\geq 2$ years of follow-up after peroral endoscopic myotomy.

\begin{tabular}{|c|c|c|c|}
\hline & $\begin{array}{l}\text { Anterior } \\
\text { POEM, n } \\
(n=54)\end{array}$ & $\begin{array}{l}\text { Posterior } \\
\text { POEM, n } \\
(n=57)\end{array}$ & $\begin{array}{l}\text { Total, } n \\
(\%) \\
(n=111)\end{array}$ \\
\hline \multicolumn{4}{|l|}{ Clinical status } \\
\hline - No change & 46 & 42 & $88(79)$ \\
\hline - Success to failure & 5 & 9 & $14(13)$ \\
\hline - Failure to success & 3 & 6 & $9(8)$ \\
\hline \multicolumn{4}{|l|}{ Dysphagia score } \\
\hline - No change & 42 & 39 & $81(73)$ \\
\hline - Worsening & 9 & 12 & $21(19)$ \\
\hline - Improvement & 3 & 6 & $9(8)$ \\
\hline \multicolumn{4}{|l|}{ GERDQ score } \\
\hline - No change & 19 & 30 & $49(44)$ \\
\hline - Worsening & 15 & 11 & $26(23)$ \\
\hline - Improvement & 20 & 16 & $36(32)$ \\
\hline \multicolumn{4}{|l|}{ PPI usage } \\
\hline - No change & 40 & 45 & $85(77)$ \\
\hline - No PPI to PPI use & 10 & 7 & $17(15)$ \\
\hline - PPI use to no PPI & 4 & 5 & $9(8)$ \\
\hline
\end{tabular}

$(P=0.08)$, and $19 / 22(86 \%)$ to $14 / 19(74 \%)$ in patients with achalasia type III $(P=0.03)$ ( $\triangleright$ Table 3$)$. There were nonsignificant changes in dysphagia and GERDQ scores across the three achalasia subtypes from 1 year to $\geq 2$ years post-procedure. The majority of patients were off-PPI at $\geq 2$ years in all three achalasia subtypes $(P=0.72)$ ( $\vee$ Table 3$)$.

\section{Longitudinal analysis of clinical status and GERD}

Plotting the clinical success across the time intervals of 3 months, 6 months, 1 year, and 2 years (Fig. $1 \mathrm{~s}$ ) showed a nonsignificant trend of earlier drop in clinical success over time in the posterior arm compared with the anterior arm (OR 0.5, 95 $\% \mathrm{Cl} 0.12-1.03 ; P=0.04)$. There was a nonsignificant change in median GERDQ score across baseline and post-POEM time intervals between both arms (Fig. $2 \mathrm{~s}$ ).

\section{Discussion}

POEM continues to gain popularity among gastroenterologists and surgeons as a nonsurgical treatment for achalasia. Defining the optimal technical approach to achieve durable clinical success with the lowest rate of post-POEM GERD is of paramount importance. In this regard, it is necessary to answer the question regarding which myotomy orientation would ultimately be associated with a better long-term benefit. It has been post- 
- Table 3 Post-procedure outcomes at 1 and $\geq 2$ years after peroral endoscopic myotomy by achalasia subtype.

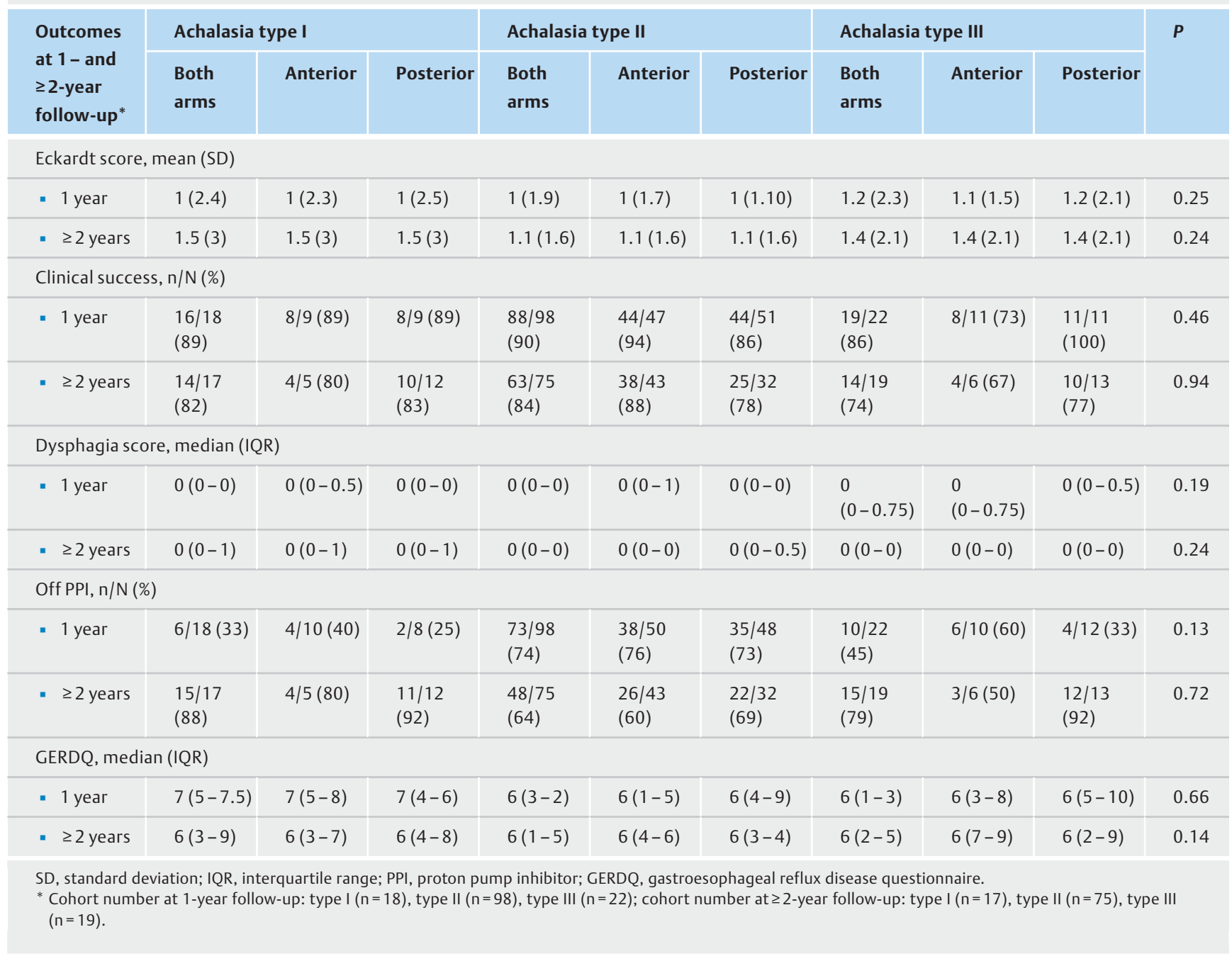

ulated that the anterior approach ( 1 to 2 o'clock position) preserves the oblique muscle fibers, thus maintaining the integrity of the angle of His. This, in turn, might theoretically result in a lower risk of post-myotomy GERD. Alternatively, the posterior approach ( 5 to 6 o'clock position) carries a lower risk of bleeding as it avoids the branches of the left gastric artery during submucosal tunneling and myotomy. Despite limited prior evidence supporting the above hypotheses $[9,10]$, results from our initial RCT [7] did not provide support for these assumptions. The rate of GERD measured by abnormal acid exposure, GERDQ score, and PPI use, as well as the rate of adverse events (including bleeding) were not significantly different between the two groups.

In an effort to report long-term results, post-trial follow-up was performed in this study. The overall clinical success was $82 \%$ at $\geq 2$ years compared with $89 \%$ at 1 year post-POEM. The overall drop in the rate of clinical success was consistent with results of the recent RCT by Werner et al. [4], which reported a clinical success rate of $83 \%$ at 2 years post-POEM. Moreover, there was no significant difference in clinical suc- cess between the anterior ( $85 \%)$ and posterior $(79 \%)$ treatment arms at $\geq 2$ years post-POEM.

As the development of post-POEM GERD continues to remain a concern because of its potential long-term effects [11, 12], as well as a possible higher rate of GERD associated with POEM compared with laparoscopic Heller myotomy [13], determining the approach associated with a lower rate of post-POEM GERD is crucial. The median GERDQ score was similar in both study arms, with no increase compared with 1 year. However, there was a significant increase in PPI use in the anterior group ( $41 \%$ at $\geq 2$ years compared with $28 \%$ at 1 year) and a nonsignificant $5 \%$ decrease in PPI use in the posterior group. Although no strong conclusions can be drawn, it is reasonable to state that the anterior approach did not perform better in terms of lower reflux rate post-POEM.

Available comparative outcome data between the anterior and posterior techniques are limited to three randomized trials with a follow-up time of up to 1 year post-POEM $[7,9,10]$. Overall, clinical efficacy is believed to be equivalent during shortterm follow-up. Ramchandani et al. [9] reported higher esoph- 
ageal acid exposure in the posterior myotomy group. Two meta-analyses [5,6] have established the comparable shortterm clinical efficacy and safety of both approaches. The current study contributes comparative data between both approaches during medium-term follow-up.

The study has several limitations. Post-POEM follow-up at $\geq 2$ years was based entirely on subjective assessment tools. A limited number of patients underwent post-trial upper endoscopy or objective clinical assessments (high-resolution esophageal manometry and/or objective $\mathrm{pH}$ testing) (Table $3 \mathbf{s}$ ). Using the Eckardt score as the only indicator for clinical success has its limitations, although it is still a widely accepted scoring system in achalasia trials [14]. Similarly, the use of GERDQ for GERD assessment, as opposed to objective $\mathrm{pH}$ testing, does not account for asymptomatic GERD, which was reported to be $60.1 \%$ in a multicenter cohort of 282 patients post-POEM [15]. Conversely, GERDQ has limited specificity in terms of differentiating GERD from symptoms related to post-POEM clinical failure. The dropout rate between 1 and $\geq 2$ years was $20 \%$ and the baseline patient characteristics, notably achalasia type, were not controlled between the two arms, which could potentially have introduced selection bias (Table 4s). Additionally, noninferiority was not detected at $\geq 2$ years post-POEM due to the lack of adequate power. However, the strength of this study is that it presents the long-term outcomes of a prospectively maintained cohort with initially controlled randomized arms.

In conclusion, the anterior and posterior POEM techniques remained equally effective at $\geq 2$ years and decreases in efficacy were similar between the two approaches over time. GERD outcomes were also similar between both groups during mediumterm follow-up.

\section{Competing interests}

Professor Khashab is a consultant for Boston Scientific, Olympus, and Medtronic, and a medical advisory board member for Boston Scientific and Olympus. Dr. Pioche has received fees from Norgine, and fees and nonfinancial support from Olympus and Boston Scientific. All other authors declare that they have no conflicts of interest.

\section{References}

[1] Kahrilas PJ, Katzka D, Richter JE. Clinical practice update: the use of per-oral endoscopic myotomy in achalasia: expert review and best practice advice from the AGA Institute. Gastroenterology 2017; 153 : 1205-1211

[2] Zaninotto G, Bennett C, Boeckxstaens G et al. The 2018 ISDE achalasia guidelines. Dis Esophagus 2018; 31: doi:10.1093/dote/doy071

[3] Khashab MA, Vela MF, Thosani $\mathrm{N}$ et al. ASGE guideline on the management of achalasia. Gastrointest Endosc 2020; 91: 213-227.e6

[4] Werner YB, Hakanson B, Martinek J et al. Endoscopic or surgical myotomy in patients with idiopathic achalasia. N Engl J Med 2019; 381: $2219-2229$

[5] Rodriguez de Santiago E, Mohammed N, Manolakis A et al. Anterior versus posterior myotomy during poem for the treatment of achalasia: systematic review and meta-analysis of randomized clinical trials. J Gastrointestin Liver Dis 2019; 28: 107-115

[6] Mohan BP, Ofosu A, Chandan S et al. Anterior versus posterior approach in peroral endoscopic myotomy (POEM): a systematic review and meta-analysis. Endoscopy 2020; 52: 251-258

[7] Khashab MA, Sanaei O, Rivory J et al. Peroral endoscopic myotomy: anterior versus posterior approach: a randomized single-blinded clinical trial. Gastrointest Endosc 2020; 91: 288-297.e7

[8] Jones R, Junghard O, Dent J et al. Development of the GerdQ, a tool for the diagnosis and management of gastro-oesophageal reflux disease in primary care. Aliment Pharmacol Ther 2009; 30: 1030-1038

[9] Ramchandani M, Nabi Z, Reddy DN et al. Outcomes of anterior myotomy versus posterior myotomy during POEM: a randomized pilot study. Endosc Int Open 2018; 6: E190-198

[10] Tan Y, Lv L, Wang X et al. Efficacy of anterior versus posterior per-oral endoscopic myotomy for treating achalasia: a randomized, prospective study. Gastrointest Endosc 2018; 88: 46-54

[11] Rösch T, Repici A, Boeckxstaens G. Will reflux kill POEM? Endoscopy 2017; 49: 625-628

[12] Ichkhanian Y, Benias P, Khashab MA. Case of early Barrett cancer following peroral endoscopic myotomy. Gut 2019; 68: 2107-2110

[13] Werner YB, Costamagna G, Swanstrom LL et al. Clinical response to peroral endoscopic myotomy in patients with idiopathic achalasia at a minimum follow-up of 2 years. Gut 2016; 65: 899-906

[14] Taft TH, Carlson DA, Triggs J et al. Evaluating the reliability and construct validity of the Eckardt Symptom Score as a measure of achalasia severity. Neurogastroenterol Motil 2018; 30: e13287

[15] Kumbhari V, Familiari P, Bjerregaard NC et al. Gastroesophageal reflux after peroral endoscopic myotomy: a multicenter case-control study. Endoscopy 2017; 49: 634-642 